\title{
La Investigación Basada en Diseño en Tecnología Educativa
}

\section{Design-Based Research in Educational Technology}

\author{
Bárbara de Benito Crosetti \\ Universidad de las Islas Baleares \\ barbara.debenito@uib.es \\ Jesús María Salinas Ibáñez \\ Universidad de las Islas Baleares \\ jesus.salinas@uib.es
}

Recibido: 08/06/2016

Aceptado: 21/06/2016

Publicado: 30/06/2016

\section{RESUMEN}

Se entiende por Investigación Basada en Diseño un tipo de investigación orientado hacia la innovación educativa cuya característica fundamental consiste en la introducción de un elemento nuevo para transformar una situación. Este tipo de investigación trata de responder a problemas detectados en la realidad educativa recurriendo a teorías científicas o modelos disponibles de cara a proponer posibles soluciones a dichos problemas. A este fin, se diseñan programas, paquetes didácticos, materiales, estrategias didácticas, etc.., que se someten a pruebas y validación, y, una vez mejorados, se difunden a la realidad escolar. El proceso de investigación presenta, generalmente, dos etapas: investigar hasta crear un nuevo producto y sus sucesivas mejoras, y por otro lado aportar conocimiento en forma de principios que contribuyen a nuevos procesos de diseño. Entendiendo producto no sólo objetos materiales (libros de texto, programas de vídeo, aplicaciones de ordenador, juegos de simulación,), sino también procesos y procedimientos (métodos de enseñanza, planes de organización escolar, estrategias didácticas, distintos programas...).

\section{PALABRAS CLAVE}

Metodología de investigación; investigación basada en diseño; tecnología educativa.

\section{ABSTRACT}

The Design-Based Research is understood as a kind of educational innovation oriented research whose fundamental feature is the introduction of a new element to transform a situation. This type of research seeks to address problems identified in the educational reality using scientific theories or models available face to propose possible solutions to these problems. For this purpose, programs, training packages, materials, teaching strategies, etc., are designed, tested and validated, and once improved, spread to the school reality. The research process has generally two ap: research to create a new product and its successive improvements, and on the other hand provide knowledge in the form of principles that contribute to new design processes. Understanding product not only material objects (textbooks, video programs, computer applications, simulation games, ...) but also processes and procedures (teaching methods, school organization plans, teaching strategies, different programs...).

\section{KEYWORDS}

Research methodology; design-based research; educational technology.

\section{CITA RECOMENDADA}

De Benito, B. y Salinas, J.M. (Año). La investigación basada en diseño en Tecnología Educativa. RIITE. Revista Interuniversitaria de Investigación en Tecnología Educativa, 0, 44-59. Doi: http://dx.doi.org/10.6018/riite/2016/260631 


\section{INTRODUCCIÓN}

La agenda de investigación en el campo de la Tecnología Educativa (TE) está caracterizada por experimentar un ritmo acelerado en las novedades y temas emergentes, por ofrecer un gran abanico de elementos de interés y por estar fuertemente influenciada por las modas. En este contexto, puede que no se esté prestando la debida atención al valor y al rigor de las investigaciones relacionadas con el uso y las posibilidades de las TIC en los procesos educativos.

La investigación en Tecnología Educativa, lo mismo que ocurre con la investigación en el campo educativo en general, debería orientarse a lograr impacto real en las políticas educativas, a avanzar en el conocimiento de cómo ocurre el aprendizaje en los nuevos escenarios de aprendizaje, a estudiar los cambios que ocurren en las prácticas, a solucionar problemas educativos y proporcionar pautas y recursos a los profesionales implicados en la práctica, entre otras (Salinas, 2012).

Si consideramos la educación y con ella la TE como una ciencia con un fuerte componente de diseño, enfatizando su orientación interdisciplinar y orientada a la resolución de problemas, una opción metodológica válida y cada vez con mayor implantación la constituye la investigación basada en diseño (IBD, en adelante). Se entiende aquí diseño, en la acepción de Jarvinen (2001), como la transformación desde una situación conocida, considerada problemática por alguna de las partes interesadas, a una más deseable.

De acuerdo con Reeves (2006), la investigación relacionada con las TIC en educación se alinea con alguno de los paradigmas positivista, interpretavista, crítico, heurístico y de diseño que dicho autor señala. Pero sin duda el paradigma de la investigación basada en diseño inspira de forma preferente gran parte de la investigación relacionada con el e-learning y con los escenarios virtuales de aprendizaje.

\section{DEFINICIÓN DE LOS ESTUDIOS DE DISEÑO}

\subsection{Origen}

Este tipo de investigaciones, que tiene su campo de aplicación en diferentes ciencias aplicadas, ha sido utilizada en educación desde hace mucho tiempo. Cabe decir que el interés por la misma ha resurgido a principio de los 90, sobre todo por los trabajos de diSessa, (1991), Brown (1992) y Collins (1992) y su énfasis está en la producción del conocimiento con el objetivo último de mejorar los procesos del diseño educativo, desarrollo y evaluación (Richey y Nelson, 1996; Richey, Klein y Nelson, 2003). Sus raíces, sin embargo, están en el campo de la ingeniería y otras ciencias aplicadas.

De hecho, este paradigma surge, según Anderson (2005), de la falta de impacto de la investigación educativa en el sistema educativo y se centra en el desarrollo y evaluación sistemáticos y multifacéticos de intervenciones en contextos educativos auténticos (en oposición a la investigación conducida en condiciones de laboratorio). De forma parecida a la investigación-acción, la investigación basada en diseño se ocupa de problemas reales que son identificados por los profesionales en la práctica.

En este sentido, Burkhardt y Schoenfeld (2003) ya afirmaban la existencia de una gran distancia entre los métodos tradicionales de investigación utilizados y la práctica educativa. Basan su argumento en tres puntos fundamentales:

- La investigación educativa generalmente no está orientada a producir avances en la práctica, aunque suele proporcionar información interesante. Según estos autores, resultaría más útil si la estructura y organización de las investigaciones estuvieran más relacionadas con las necesidades prácticas del sistema educativo. 
- Los estudios de desarrollo sobre el diseño de instrumentos y procesos, desde el punto de vista de la implementación son muy escasos en el ámbito de la educación.

- Se requieren nuevas formas de trabajar y mayor coordinación entre la investigación, diseño, desarrollo, políticas y práctica.

No se trata tanto de una investigación empírica, cuantitativa orientada a la generalización de resultados, sino de una investigación con implicaciones sobre la práctica, cuyo énfasis es la solución de problemas y la construcción de conocimiento dirigido al diseño, desarrollo y evaluación del proceso educativo, así como a desarrollar principios y orientaciones para futuras investigaciones y que cada día acumula mayor respaldo (Cabero, 2004; Driscoll y Dick, 1999; Martínez, 1994).

Este tipo de investigaciones pretende responder a lo que Stokes (1997) llamó "investigación básica inspirada en el uso", en la línea de lo que demandan Burkhardt y Schoenfeld (2003) y que es distinta tanto de la investigación básica pura como de la aplicada.

Por lo tanto, este nuevo planteamiento surge de la necesidad de aplicar los resultados de la investigación a la práctica y al desarrollo de la teoría, enfocados ambos a la resolución de problemas prácticos.

\subsection{Conceptualización}

Este tipo de "investigación básica inspirada en el uso" ha sido etiquetada por los investigadores de la educación de distintas formas, siempre para referirse a distintos acercamientos a la investigación que se relacionan con el trabajo del diseño y de desarrollo (Barab y Squire, 2004; Brown, 1992; Collins, 1992; diSessa, 1991; Lee y Reigeluth, 2003; Reeves, 2000; Reigeluth y Frick, 1999; van den Akker y Plomb, 1993; van den Akker, 1999).

Hay diversos conceptos en la literatura para denominarla: Estudios de diseño; experimentos de diseño (Brown, 1992; Collins, 1992); investigación en diseño (Bannan-Ritland, 2003; Design-Based Research Collective, 2003; Kelly, 2003; Wang y Hannafin, 2005), investigación de desarrollo (van den Akker, 1999), investigación formativa (Reigeluth y Frick, 1999; Walker, 1992;), evaluación formativa, investigación de ingeniería educativa, investigación basada en diseño, investigación de diseño educativo (McKenney y Reeves, 2012, 2014).

Wang y Hannafin (2005, p.6), por su parte, la definen como "una metodología sistemática, pero flexible, dirigida a la mejora de la práctica educativa mediante análisis, diseño, desarrollo e implementación iterativos, basados en la colaboración de investigadores y practicantes en un entorno real y que persigue principios de diseño y teoría basadas en contexto".

Para Plomp (2010) la IBD viene a ser "el estudio sistemático de diseñar, desarrollar y evaluar intervenciones educativas (ya sean programas, estrategias o los materiales de enseñanza-aprendizaje, productos y sistemas) como soluciones a problemas complejos de la práctica educativa, que al mismo tiempo tiene por objeto la mejora de nuestro conocimiento sobre las características de estas intervenciones y sobre los procesos de diseño y desarrollo de las mismas" (p.13).

Sobre su aplicación en la educación, Van den Akker (1999) identifica cuatro sub-dominios del campo educativo donde la IBD tiene ya cierta tradición: Currículum, Medios y Tecnología, Instrucción y Aprendizaje, y Didáctica y formación de profesores. A pesar de que en los subdominios de instrucción y aprendizaje o didáctica y formación de profesores este tipo de investigación ha tenido gran desarrollo, es en Medios y Tecnología, al ser un área de rápido crecimiento donde tiene un lugar prominente.

Por su parte, Richey y Nelson (1996), Richey, Klein y Nelson (2003) proporcionan la descripción y el análisis más comprensivos de la "investigación de desarrollo" en el subdominio de la T.E. hasta ahora. Mencionan como su última meta: mejorar los procesos del diseño educativo, el desarrollo, y la evaluación, basados tanto en la resolución de problemas específicos y muy contextualizados, como en procedimientos generalizados de la investigación. 
Estos autores hacen una distinción clara entre el estudio del proceso y la ejecución de ese proceso:

a) En el primer caso, estas investigaciones son utilizadas tanto para el contraste de teorías, como para la elaboración de modelos y principios. Uno de los roles que el diseño ha desarrollado en la investigación educativa ha sido actuar como una estrategia para contrastar teorías.

Podemos decir que en la IBD los estudios son llevados a cabo para desarrollar teorías, no solamente para ajustar y probar "aquello que funciona" (Van den Akker, 1999), aunque, como señalan Cobb y otros (2003) estas teorías son relativamente humildes al dirigirse a dominios específicos del proceso de enseñanza-aprendizaje y a respaldar actividades de diseño. Se trata, por tanto, de teorías para el mundo real más que de grandes teorías del aprendizaje que, en algunos casos, pueden presentar dificultades para proyectarse en unas circunstancias particulares. Para Edelson (2002) pueden ser de tres tipos: teorías de dominio, marcos de referencia para el diseño y metodologías de diseño.

b) En el segundo caso, se trata de procesos de innovación, de mejora de la práctica.

Para Shavelson, Phillips, Towne y Feuer (2003), este tipo de estudios son también apropiados para contrastar teorías en la práctica. Pero para estos autores además lo son para trabajar de forma colegiada con los profesionales de la práctica, co-construyendo conocimiento; para confrontar los problemas cotidianos que afectan a la enseñanza y aprendizaje -en la clase, en la escuela o en una comunidad- y adaptar la enseñanza a estas particulares condiciones; para el reconocimiento de los límites de la teoría; y para capturar lo específico de la práctica y las ventajas potenciales de la adaptación y afinación iterativos de las teorías en dicho contexto. En resumen, tienden a conducir entornos educativos innovadores (Brown, 1992).

Para el Design-Based Research Collective (2003), se centra en diseñar y explorar el rango completo de innovaciones: tanto artefactos, como aspectos menos concretos tales como estructura de la actividad, instituciones, sistemas de apoyo, curricula... Para este colectivo la IBD va más allá de diseñar y contrastar intervenciones particulares. Las intervenciones incorporan demandas teóricas específicas sobre la enseñanza y el aprendizaje y reflejan un entendimiento acerca de las relaciones entre teoría, artefactos diseñados y práctica. Al mismo tiempo, la investigación puede contribuir al avance mismo de las teorías de la enseñanza y el aprendizaje.

Pueden encontrarse diversidad de actividades de investigación -en nuestro caso relacionadas con la Tecnología Educativa- bajo el paraguas de IBD. También se puede distinguir, a otro nivel de abstracción, una meta general común a todos los enfoques: reducir la incertidumbre en la toma de decisiones y en el diseño y desarrollo de intervenciones educativas. El término intervención sirve de denominador común para productos, programas, materiales, procedimientos, escenarios, procesos y otros similares.

Estas investigaciones parten de una comprensión amplia de una 'ecología del aprendizaje', por el diseño de sus elementos y por el anticipo de cómo estos elementos funcionan en conjunto para favorecer el aprendizaje. Se utiliza la metáfora de la ecología para enfatizar que los contextos diseñados son conceptualizados como sistemas interactivos enfocados a la complejidad del escenario educativo, en lugar de una colección de actividades de una lista separada de factores que influyen en el aprendizaje.

Otro aspecto importante, desde el punto de vista metodológico y en relación a la posible generalización de los conocimientos alcanzados, lo constituye la validez de este tipo de investigación. Reigeluth y Frick (1999) proponen tres dimensiones: Efectividad, eficiencia y significado. Cada una de estas dimensiones se presenta de distinta forma en cada situación, pero es conveniente considerarlas en la IBD de cara a su validez y generalización.

El valor de la IBD debe ser medida, en todo caso, por su habilidad para mejorar la práctica educativa, para mejorar la intervención, y en especial las intervenciones asociadas a procesos de innovación que suelen darse en el campo de la Tecnología Educativa. El Design-Based 
Research Collective (2003) señala 4 áreas en las que los métodos de investigación basada en diseño presentan mayores perspectivas:

- Explorar las posibilidades para crear entornos nuevos de enseñanza-aprendizaje.

- Desarrollar teorías de instrucción y aprendizaje basadas en el contexto.

- Avanzar y consolidar el conocimiento sobre diseño didáctico.

- Incrementar nuestra capacidad para la innovación educativa.

\section{CARACTERÍSTICAS DE LA INVESTIGACIÓN BASADA EN DISEÑO}

Las características de la IBD pueden definirse siguiendo a Brown (1992), Collins (1992) y Reeves, Herrington y Oliver (2002) de la siguiente forma:

- Centradas en amplios problemas complejos en contextos reales.

- Implica colaboración intensiva entre investigadores y practicantes.

- integrar principios de diseño reconocidos e hipotéticos con las potencialidades tecnológicas para proporcionar soluciones realizables a estos problemas complejos.

- Poner en marcha estudios rigurosos y reflexivos para probar y refinar entornos de aprendizaje innovadores, así como para definir nuevos principios de diseño.

- Requiere implicación a largo plazo que permita el refinamiento continuado de protocolos y cuestiones.

- Mantiene un compromiso tanto con la construcción y ampliación teórica, como con la resolución de problemas del mundo real.

Wang y Hannafin (2005) resumen sus características en 5: pragmática; fundamentada; interactiva, iteractiva y flexible; integrada; contextual.

Hay que considerar la IBD desde los principios que se mencionaron: ser recursiva (iterativa), reflexiva y participativa.

- La iteración, en efecto, supone un proceso de diseño y desarrollo que permite tanto a los usuarios como a los expertos participar completamente del proceso de revisión y reformulación.

- Al ser reflexiva, se opone al enfoque de la racionalidad técnica y asume que muchos, si no la mayoría, de los problemas importantes en la práctica profesional no pueden definirse y resolverse con soluciones preconcebidas.

- Al ser participativa, refleja el cambio de perspectiva de considerar al experto, a uno en el que el diseñador e investigador son parte de un equipo.

Por su parte Cobb y otros (2003) la caracterizan como iteractiva, centrada en procesos, intervencionista, colaborativa, multinivel, orientada a la utilidad, y fundamentada en la teoría.

Otro aspecto que caracteriza a la IBD es ser inconclusa, inacabada, abierta (Hoadley, 2002). Esto representa una ventaja para la investigación relacionada con la Tecnología Educativa, dado que sus procesos de diseño se adaptan bien a cuestiones abiertas de las investigaciones. 


\section{APLICACIÓN}

Pueden mencionarse distintos motivos para la iniciación y puesta en marcha de IBD. Primero, los enfoques tradicionales con su foco en el conocimiento descriptivo, proporcionan pocas especificaciones y soluciones útiles para el diseño y desarrollo de una variedad de problemas de educación. El desafío más grande para los diseñadores profesionales es probablemente cómo hacer frente a las múltiples incertidumbres en sus tareas complejas y en contextos muy dinámicos. Si buscan la ayuda de la investigación para reducir esas incertidumbres, suelen enfrentarse a frustraciones de diversa índole: las respuestas son demasiado cercanas para ser significativas, demasiado superficiales para ser instrumentales, demasiado artificiales para ser relevantes, y con frecuencia, llegan demasiado tarde para ser aplicables.

\subsection{Fases y estructura de la investigación}

Los procesos de investigación realizados bajo este enfoque metodológico no están bien definidos. Sin embargo, independientemente del número de etapas en que se divida el proceso, todos ellos incluyen una serie de acciones comunes como son: definición del problema, diseño, desarrollo, implementación y evaluación.

Entre las distintas propuestas en relación a la estructura de la investigación, quizá el modelo propuesto por Reeves (2000; 2006) sea el más representativo (Fig. 1). En él, la investigación se inicia con el análisis de la situación y la definición del problema. Las posibles soluciones se diseñan a partir de un marco teórico de referencia, de ahí la importancia de la evaluación y revisión, que incide tanto sobre la fundamentación teórica como sobre los puntos positivos y negativos de la intervención. La fase siguiente es la implementación seguida de la recogida de información (validación según el esquema), esta última se lleva a cabo a lo largo de todo el proceso. El proceso de investigación se concreta mediante ciclos continuos de diseño, validación, análisis y rediseño, conduciendo las diferentes iteraciones a la mejora del cuerpo teórico y el perfeccionamiento de la intervención.

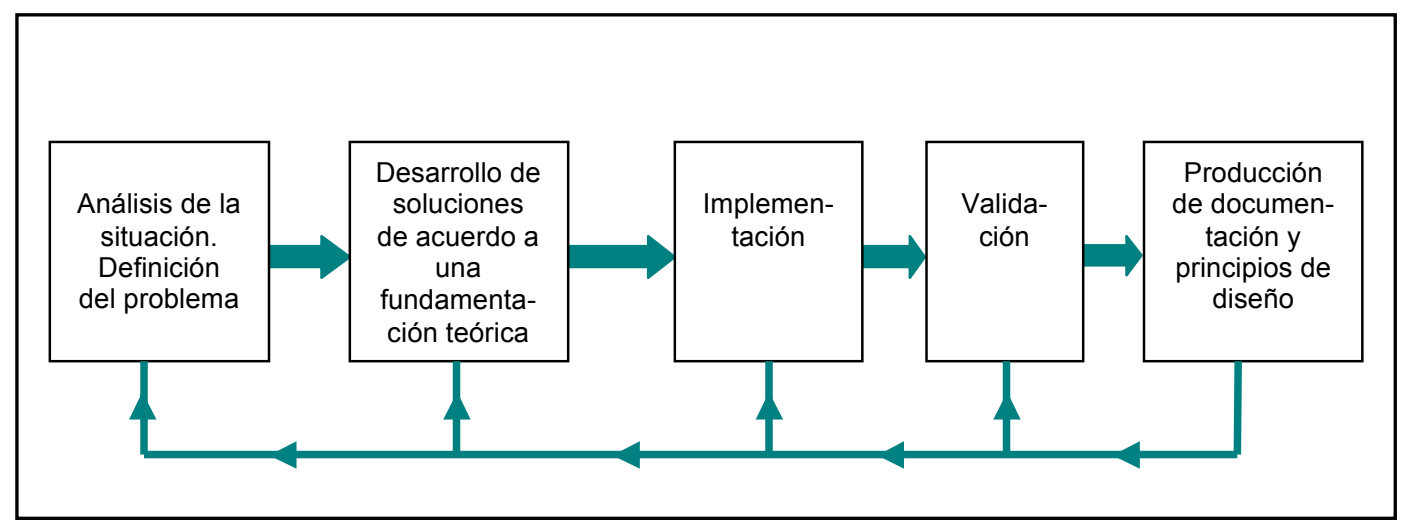

Figura 1. Proceso de la investigación de desarrollo (adaptado de Reeves, 2000 en de Benito,

2006)

Garello, Rinaudo y Donolo (2010), por su parte, señalan la existencia de tres fases centrales: fase de preparación del diseño, fase de implementación y fase de análisis retrospectivo. En cada una de ellas se incluyen procedimientos metodológicos particulares.

Easterday, Lewis y Gerber (2014) proponen un modelo de IBD en 6 fases: focalizar, comprender, definir, concebir, construir y probar.

Plomp (2010), señala algunas diferencias en la estructura y la organización de las fases de investigación en función de los objetivos de investigación si estos van encaminados a estudios de validación o si se trata de procesos de desarrollo.

En cualquier caso, un proyecto de este tipo puede incluir varias etapas distintas, implicando cada una informar y analizar una serie de datos. Es decir, la IBD puede estructurarse en distintas partes o componentes (Richey, Klein y Nelson, 2003; Richey y Klein, 
2014; Kelly, 2003): Se trata de subestudios que pueden conducir a analizar y definir el problema instruccional, a especificar el contenido, a determinar los instrumentos de fiabilidad y validez.

\subsection{Fuentes, métodos y productos de investigación}

Hay que señalar que la IBD no dispone de una metodología propia, sino que se apoya en cualquiera de los métodos utilizados, aunque existe cierta tendencia a utilizar métodos cualitativos. Lo que verdaderamente la caracteriza es ser participativa, colaborativa, desde el momento en que en el proceso se adopta un proyecto de grupo, por lo que el sistema de trabajo es básicamente colaborativo, tanto entre el equipo de investigadores, como entre estos y los expertos externos, en su caso.

Al caracterizarse por ser mutivariable y con foco principal en los cambios (o aprendizaje), este tipo de estudios se distingue por generar un sistema de registro de los fenómenos comprensivo. La documentación implica grabaciones de vídeo, audio entrevistas, cuestionarios, análisis de materiales, etc...

Precisamente la envergadura del sistema de registro representa una de sus posibles limitaciones al recogerse gran cantidad de datos y registros procedentes de observaciones (cualitativas y cuantitativas) de forma que muchas veces impiden un correcto análisis de los mismos.

Este tipo de investigaciones pretende estudiar la propia actividad educativa con la intención de mejorarla, y su característica fundamental reside en preocuparse por resolver los problemas concretos, en el propio contexto. La IBD trata de responder a problemas detectados en la realidad educativa recurriendo a teorías científicas o modelos disponibles en orden a ofertar posibles soluciones a los problemas (Escudero, 1984). A este fin, se diseñan programas, paquetes didácticos, materiales, etc.., se someten a pruebas y validación, y, una vez mejorados, se difunden a la realidad educativa.

Ya se señaló la importancia de la implicación de investigadores junto a los prácticos en los procesos. También los expertos son requeridos con cierta frecuencia, tanto para evaluación, para contrastar modelos y teorías, para el desarrollo de métodos para establecer la validez interna y externa, etc. (Cobb y otros, 2003; Richey y Klein, 2014; McKenney y Reeves, 2012, 2014).

El objetivo de este tipo de estudios consiste en describir un fenómeno y para ello la observación sistemática de los fenómenos, una vez producidos, es básica. Para esta observación pueden utilizarse distintas técnicas que pertenecen tanto a la metodología cuantitativa (tests, encuestas, cuestionarios, etc.) como a la cualitativa (estudios etnográficos...). La convergencia de distintos investigadores proporciona diversidad de perspectivas y en consecuencia mayor confianza sobre los datos y evidencias. Es importante la composición del grupo de investigación y el grado de experiencia que ofrece... y la relación entre investigadores y profesionales de la práctica.

\subsection{Técnicas}

Al tratarse de investigación sobre procesos en marcha y en los que el investigador se encuentra implicado, o también en los estudios reconstructivos, resulta de gran interés disponer de un buen sistema de registro. En cuanto a los instrumentos y registro de la información son habituales (Cobb, 2003; Richey y Nelson, 1996): estudio de casos; análisis de documentos y artefactos; observación participante y no participante; entrevistas estructuradas y/o semiestructuradas; diseños experimentales y cuasiexperimentales; cuestionarios diversos (dirigidos a alumnos, a diseñadores, a expertos.); test, etc.

Como se ha dicho, están caracterizados por utilizar metodologías mixtas, combinando métodos formales e informales de acuerdo a los criterios de validez (Reigeluth y Frick, 1999), y entre los principales instrumentos utilizados, podemos señalar: 
a) Análisis de documentos y artefactos, mediante bases de datos, informes técnicos, ensayo, etc.

b) Cuestionarios. Elaboración, validación y aplicación de cuestionarios diversos (dirigidos a alumnos, a diseñadores, a expertos...).

c) Estudio de casos. Se trata de casos de proceso de apoyo a grupos de alumnos que aprenden en un contexto particular proporcionando conjunto de medios, diseño del contexto de aprendizaje, etc.

d) Observación participante. El investigador recoge información a partir de su participación directa en las actividades objeto de estudio.

e) Observación no participante. El papel del investigador es de observador externo.

f) Entrevista estructurada y/o semiestructurada.

g) Análisis funcional. Basado en el razonamiento inductivo (causas consecuencias), supone la definición de objetivos o finalidades. Análisis de las funciones de las herramientas dentro del contexto o situación didáctica donde se utilizan.

h) Diseño de instrumentos y dispositivos (artefactos).

i) Panel. Seminario, grupo de expertos. Permite acceder al conocimiento, creencias y expectativas de un grupo académico que comparte una cultura común.

\subsection{Productos}

Dadas las posibilidades que ofrece este tipo de metodología, los resultados de la investigación son diversos. Si nos referimos a los resultados de la investigación en diseño y desarrollo que proponen Purao (2002) y Rossi y Sein (2003) -constructos, modelos, métodos, ilustraciones u operativización, y mejores teorías-, desde la Tecnología Educativa nos estamos ocupando de todos ellos, pero preferentemente de los tres primeros.

Tabla 1. Tipos de resultados en investigaciones basadas en diseño (Purao, 2002)

\begin{tabular}{|l|l|l|}
\hline & $\begin{array}{l}\text { Producto de } \\
\text { investigación }\end{array}$ & Descripción \\
\hline 1 & Constructo & Vocabulario conceptual del dominio \\
\hline 2 & Modelo & $\begin{array}{l}\text { Una serie de proposiciones o de declaraciones que expresan relaciones entre } \\
\text { los constructos. Representa, en este caso, el proceso, centrándose en utilidad } \\
\text { (situada) }\end{array}$ \\
\hline 3 & Método & $\begin{array}{l}\text { Serie de etapas para desarrollar una tarea (conocimiento de cómo). Planes } \\
\text { dirigidos a metas para la manipulación de constructos para lograr el modelo de } \\
\text { la solución }\end{array}$ \\
\hline 5 & Ilustración & $\begin{array}{l}\text { La operativización de constructos, modelos y métodos. Es la implementación } \\
\text { del artefacto en un entorno }\end{array}$ \\
\hline 5 & Mejores teorías & $\begin{array}{l}\text { Puede contribuir a mejorar las teorías de dos formas: } \\
- \text { Al constituirse en objeto de teorízación la construcción metodológica del } \\
\text { proceso. } \\
- \text { Al exponer relaciones entre los elementos del artefacto (falsando, } \\
\text { elaborando, confirmando relaciones previamente teorizadas...). }\end{array}$ \\
\hline
\end{tabular}

Estos resultados pueden, al mismo tiempo, asociarse a casi todos los tipos de problemas de investigación que propone Richey (1998):

- Estudio de un producto específico o del proyecto de un programa.

- Estudio de un aspecto del proceso diseño/desarrollo/evaluación.

- Desarrollo de un nuevo (o la mejora del mismo) modelo de diseño/desarrollo/evaluación.

- Validar técnicas, herramientas o modelos.

- Determinar condiciones que faciliten la implantación exitosa. 
Desde la perspectiva de la investigación de diseño educativo, donde la investigación se concibe como el desarrollo iterativo de soluciones a complejos problemas educativos que proporcionan los fundamentos de la investigación científica, las soluciones a dichos problemas pueden ser (McKenney y Reeves, 2012):

- Productos educativos (p.e. materiales multimedia; juegos de aprendizaje...).

- Procesos (p.e. estrategia de apoyo al estudiante en elearning ...).

- Programas (p.e. serie de seminarios para ayudar a profesores a desarrollar estrategias de cuestionamiento...).

- Modelos y políticas (p.e. estrategia educativa para un curso escolar, ...).

\section{ALGUNOS EJEMPLOS}

Aunque puede ser una visión parcial de cada uno de los casos que se presentan, se ha querido traer algunos ejemplos de investigaciones que responde a los tipos de resultados que proponen McKenney y Reeves (2012). Se hace énfasis en el planteamiento y estructura de la investigación, siendo conscientes de que pueden quedar descontextualizados, pero que pueden ayudar a ilustrar este tipo de investigaciones y sus posibilidades en el campo de la Tecnología Educativa.

\section{1.- Investigaciones orientadas al desarrollo de productos educativos.}

Caso 1. Diseño y validación de un instrumento de selección de herramientas para entornos virtuales basado en la toma de decisiones multicriterio (de Benito, 2006).

Se trata de un ejemplo de aplicación de la metodología de diseño para la construcción de un artefacto, en este caso un instrumento para la selección de plataformas para la creación de Entornos Virtuales de Formación.

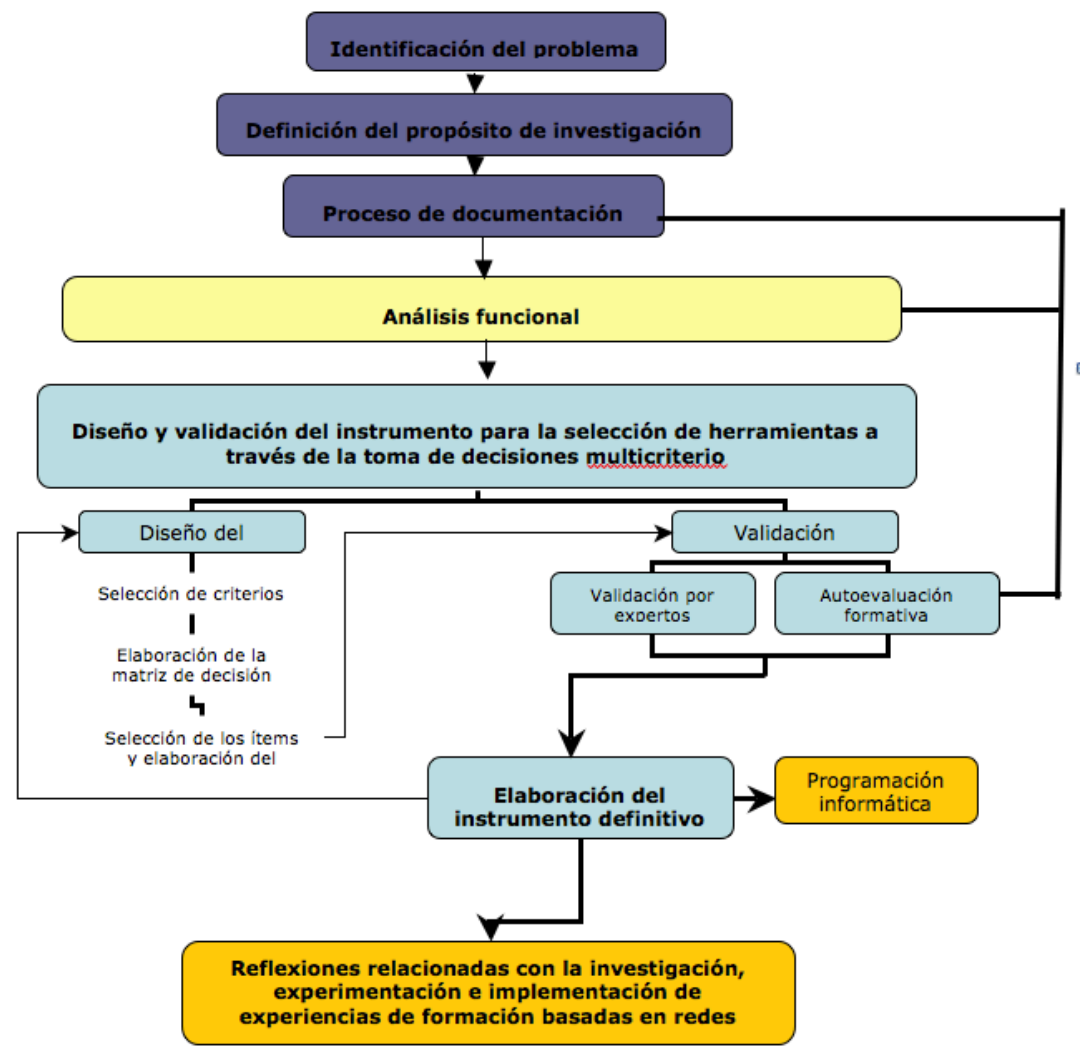

Figura 2. Fases del proceso de investigación del caso 1 


\section{2.- Investigaciones orientadas al desarrollo de procesos (principalmente estrategias didácticas mediadas por TIC).}

Caso 2. Diseño, implementación y validación de una comunidad virtual de investigadores en formación. (Moreno, 2011)

Investigación que tiene como objetivo la creación de una comunidad virtual de investigadores en formación. Se aplicó la metodología de diseño a partir de la combinación del modelo instruccional ADDIE y el de gestión de proyectos IPECC.

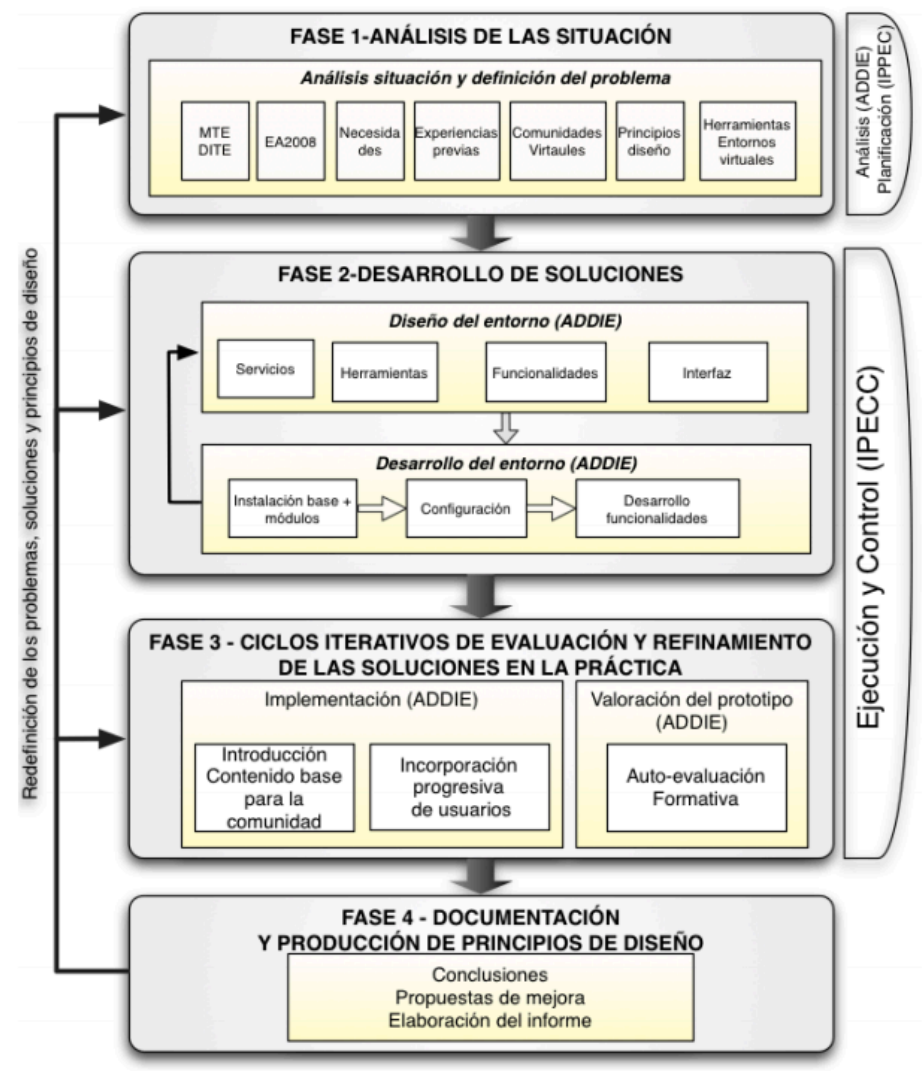

Figura 3. Fases del proceso de investigación del caso 2

Caso 3. Modelos de rediseño de acciones formativas en el entorno virtual de enseñanzaaprendizaje. Diseño y experimentación de estrategias metodológicas de integración de los entornos institucionales y abiertos. (Marín,2014)

Investigación orientada a la mejora de los procesos de enseñanza-aprendizaje en la universidad mediante el desarrollo de diferentes propuestas de estrategias metodológicas de integración didáctica de entornos institucionales de formación y de educación informal. Combina la metodología de diseño con el estudio de casos. 


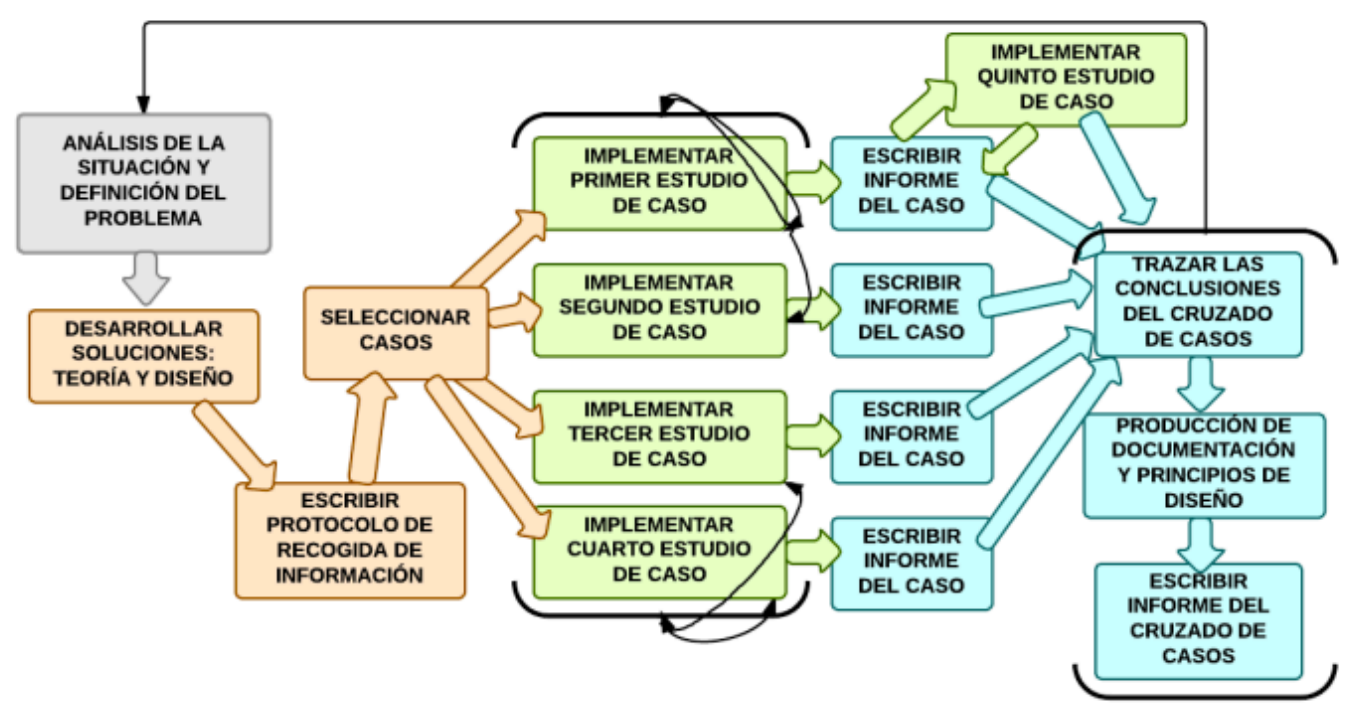

Figura 4. Fases del proceso de investigación del caso 3

Caso 4. Diseño de un procedimiento de captura y representación del conocimiento TPACK en la enseñanza universitaria. (Lizana, 2012)

La finalidad de este estudio era la construcción de un procedimiento para la captura y representación del conocimiento de docentes universitarios, con el fin de proporcionar estrategias de mejora en la transferencia y aplicación de conocimiento entre pares.

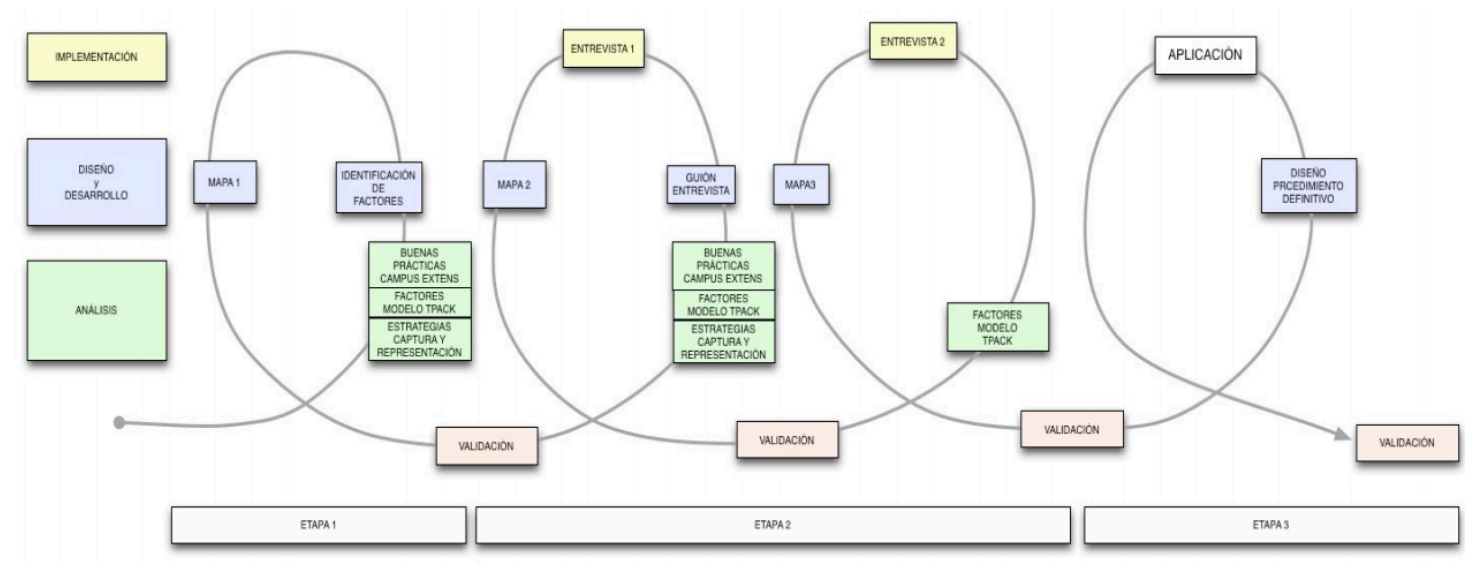

Figura 5. Fases del proceso de investigación del caso 4

\section{3.- Investigaciones orientadas al desarrollo de programas educativos.}

Caso 5. Construcción de modelos pedagógicos en entornos virtuales de aprendizaje. Propuesta institucional para la licenciatura de trabajo social en la Patagonia Austral. (Aranciaga, 2016)

La finalidad el estudio se centra en la revisión de la propuesta curricular de la Licenciatura de Trabajo Social y el modelo pedagógico en el que se imparte con la intención de incorporar propuestas metodológicas al desarrollo académico de la formación integral del Trabajador Social 


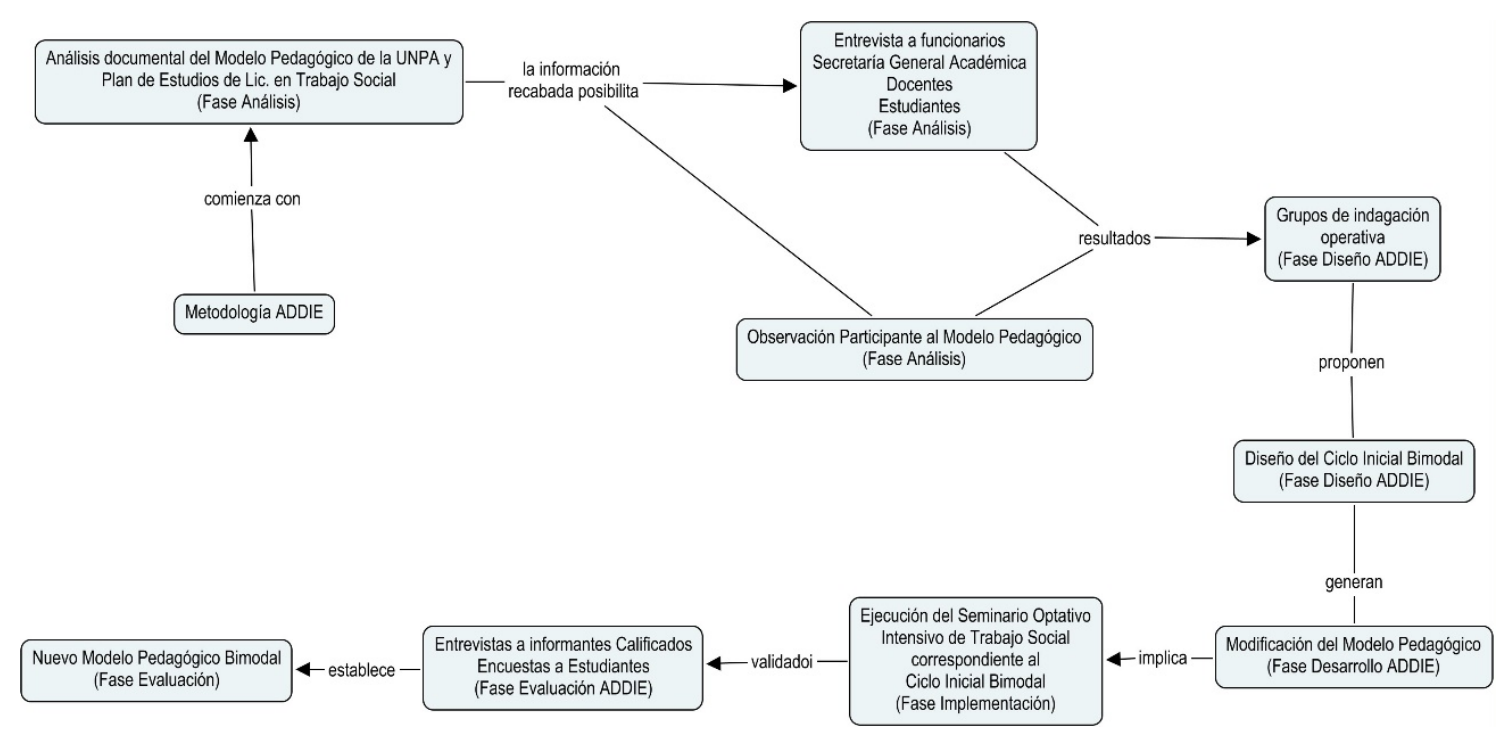

Figura 6. Fases del proceso de investigación del caso 5

\section{4.- Investigaciones orientadas al desarrollo de modelos.}

Caso 6. Organización y gestión de la tutoría online para la dirección de proyectos de investigación (Darder, 2014)

El objetivo de esta investigación era diseñar y desarrollar un procedimiento para la tutoría de proyectos de investigación on-line, ofreciendo soluciones en la praxis e identificando los elementos organizativos y procesuales para esta.

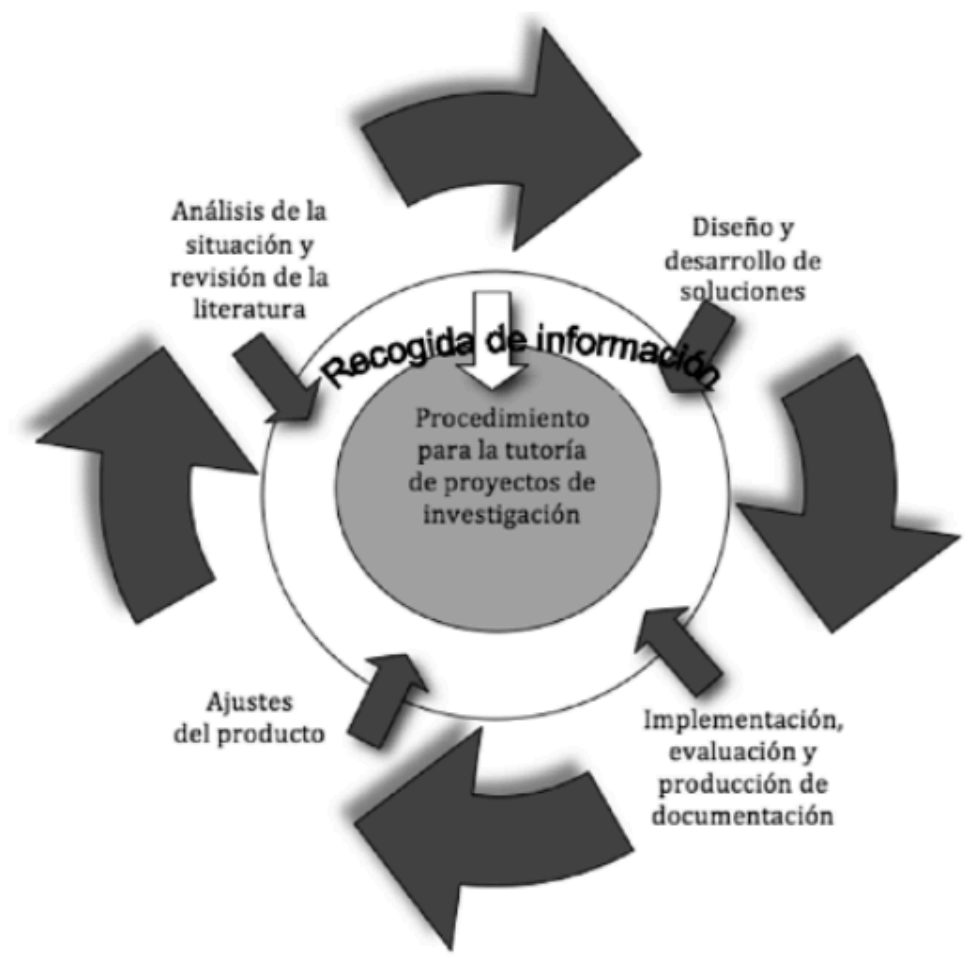

Figura 7. Fases del proceso de investigación del caso 6 


\section{REFLEXIONES FINALES}

Los estudios basados en diseño están ganando fuerza en el ámbito de la Tecnología Educativa ya que ayudan a comprender todos los pasos del proceso de creación, elaboración, revisión, implantación y diseminación, en su caso, de cualquier programa o producto relacionado con la introducción de las TIC en los procesos de enseñanza-aprendizaje.

Dan respuesta a una demanda real sobre investigación en la práctica educativa generando distinto tipo de procesos, materiales, estrategias, teorías, resultados, etc., que proveen de información de utilidad a los docentes para dar sentido a su desempeño profesional, al mismo tiempo que hacer frente a problemas complejos en la práctica educativa para los cuales no hay directrices claras ni las soluciones están disponibles.

El objetivo de este tipo de estudio no es de obtener leyes universales ni la generalización de los resultados si no de crear modelos de modos probables de andamiaje que favorezcan resultados de aprendizaje positivos. Se trata, en definitiva, del diseño y desarrollo de una intervención como una solución (innovadora) a un problema complejo, y en consecuencia el punto de partida son problemas educativos para los que no existen -o sólo se dispone de unos pocos- principios validados para estructurar y apoyar dichas actividades de diseño y desarrollo.

\section{REFERENCIAS}

Anderson, T. (2005). Design-based research and its application to a call center innovation in distance education. Canadian Journal of Learning and Technology, 31(2), 69-84. Recuperado de http://www.jofde.ca/index.php/jde/article/view/18/552

Aranciaga, I. (2015). Construcción de modelos pedagógicos en Entornos Virtuales de Aprendizaje. Propuesta institucional para la Licenciatura de Trabajo Social en la Patagonia Austral (Tesis doctoral inédita). Universitat de les Illes Balears, Palma de Mallorca.

Bannan-Ritland, B. (2003). The Role of Design in Research: The Integrative Learning Design. Framework Educational Researcher, 32(1), 21-24.

Barab, S., y Squire, K. (2004). Design-based research: Putting a stake in the ground. The Journal of the Learning Sciences, 13(1), 1-14.

Brown, A. L. (1992). Design experiments: theoretical and methodological challenges in creating complex interventions in classroom settings. Journal of the Learning Sciences, 2(2), 141178.

Burkhardt, H. y Schoenfeld, A. (2003). Improving educational research: Toward a more useful, more influential and better-funded enterprise. Educational Researcher, 32(9), 3-14.

Cabero, J. (2004). La investigación en Tecnologías de la Educación. Bordón 56(3-4), 617-634.

Cobb, P., Confrey, J., diSessa, A., Lehrer, R., y Schauble, L. (2003). Design experiments in educational research. Educational Researcher, 32(1), 9-13.

Collins, A. (1992). Toward a design science of education. En E. Scanlon y T. O'Shea (Eds.), New directions in educational technology (pp. 15-22). Berlin: Springer-Verlag.

Darder. A (2014). Organización y gestión de la tutoría online para la dirección de proyectos de investigación (Tesis doctoral inédita). Universitat de les Illes Balears, Palma de Mallorca.

De Benito, B. (2006). Diseño y validación de un instrumento de selección de herramientas para entornos virtuales basado en la toma de decisiones multicriterio (Tesis doctoral inédita). Universitat de les Illes Balears, Palma de Mallorca.

Design-Based Research Collective. (2003). Design-Based Research: An Emerging Paradigm for Educational Inquiry. Educational Researcher, 32(1), 5-8. 
diSessa, A. A. (1991). Local sciences: Viewing the design of human-computer systems as cognitive science. En J.M. Carroll (Ed.), Designing interaction: Psychology at the humancomputer interface (pp. 162-202). Cambridge, England: Cambridge University Press.

Driscoll, M.P. y Dick, W. (1999). New research paradigms in instructional technology. An inquiry. Educational Technology Research \& Development, 47(2), 7-18.

Easterday, M., Lewis, D. y Gerber, E (2014). Design-Based Research Process: Problems, Phases and Applications. ICLS Proceedings Volume I. (317-324).

Edelson, D. C. (2002). Design research: What we learn when we engage in design. Journal of the Learning Sciences, 11(1), 105-121.

Elliot, J. (1990). La investigación-acción en educación. Madrid: Morata.

Escudero, J.M. (1984). La renovación pedagógica: algunas perspectivas teóricas y prácticas. En Escudero, J.M.; González, M.T. (Eds.), La renovación pedagógica: algunos modelos teóricos y el papel del profesor. (p. 15-92) Madrid: Escuela Española.

Garello, M; Rinaudo, M. y Donolo, D. (2011). Valoración de los Estudios de diseño como metodología innovadora en una investigación acerca de la construcción del conocimiento en la universidad. RED-DUSC. Revista de Educación a Distancia-Docencia Universitaria en la Sociedad del Conocimiento. Recuperado de http://www.um.es/ead/reddusc/5

Hoadley, C. (2002). Creating context: Design-based research in creating and understanding CSCL. En G. Stahl (Ed.), Computer support for collaborative learning 2002 (pp. 453-462). Mahwah, NJ: Lawrence Erlbaum Associates, Inc.

Järvinen P. (2001). On research methods. Opinpajan kirja, Tampere.

Kelly, A. E. (Ed.) (2003). Special issue on the role of design in educational research [Special issue]. Educational Researcher, 32(1).

Lee, J. Y., y Reigeluth, C. M. (2003). Formative research on the Heuristic Task Analysis(HTA) process. Educational Technology, Research and Development, 514), 5-17

Lizana, A. (2012). Diseño de un procedimiento de captura y representación del conocimiento TPACK en la enseñanza universitaria. Proyecto fin de Master de la Universidad de Islas Baleares. Recuperado http://gte.uib.es/pape/gte/sites/gte.uib.es.pape.gte/files/files/documentos_biblio/Alexandra_Li zana_proyecto.pdf

March, S. T., y Smith, G. F. (1995). Design and natural science research on information technology. Decision Support Systems, 15(4), 251-266.

Marín, V. I. (2014). Modelos de rediseño de acciones formativas en el entorno virtual de enseñanza-aprendizaje. Diseño y experimentación de estrategias metodológicas de integración de los entornos institucionales y abiertos (Tesis doctoral inédita). Universitat de les Illes Balears, Palma de Mallorca.

Martínez, F. (1994). Investigación y nuevas tecnologías de la comunicación en la enseñanza: el futuro inmediato. Pixel-Bit. Revista de medios y educación, 2, 3-17.

McKenney, S.E. y Reeves, T. (2012). Conducting Educational Design Research. NY: Routledge.

McVay Lynch, M. y Roecker,J. (2007). Project managing E-learning. A Handbook for succesful design, delivery and management. London:Routledge. 
Moreno García, J. (2011). Diseño, desarrollo e implementación de un prototipo de entorno virtual para una comunidad de investigadores en formación. Proyecto fin de Master de la Universidad de Islas Baleares. Recuperado de http://gte.uib.es/pape/gte/sites/gte.uib.es.pape.gte/files/PFM-Juan-Moreno.pdf.

Plomp, T. (2010): Educational Design Research: An Introduction En Tjeerd Plomp y Nienke Nieveen (Ed), An Introduction to Educational Design Research Proceedings of the seminar conducted at the East China Normal University, Shanghai (PR China).

Purao, S. (2002). Design Research in the Technology of Information Systems: Truth or Dare. Working Paper. Atlanta, GA: GSU Department of CIS.

Reeves, T. C. (2000). Enhancing the Worth of Instructional Technology Research through "Design Experiments" and Other Development Research Strategies. International Perspectives on Instructional Technology Research for the 21st Century Symposium. New Orleans, LA, USA.

Reeves, T. C. (2006). Design research from the technology perspective. En J. van den Akker, K. Gravemeijer, S. McKenney, y N. Nieveen (Ed.), Educational design research (pp. 86109). London: Routledge.

Reeves, T. C., Herrington, J., y Oliver, R. (2002). Authentic activities and online learning. En J. Goody, J. Herrington y M. Northcote (Ed.), Quality conversations: Research and Development in Higher Education (Vol. 25, pp. 562-567): ACT: HERDSA.

Reeves, T., Herrington, J., y Oliver, R. (2005). Design research: A socially responsible approach to instructional technology research in higher education. Journal of Computing in Higher Education, 16(2), 97-116.

Reigeluth, C. y Frick, T. (1999). Formative research: A methology for Creating and Improving Design Theories. En Reigeluth, C. (Ed.). Instructional-Design Teheories and Models. A New Paradigm of Instructional Theory (Vol. II) 633-652. Mahwah (NJ), USA:Lawrence Erlbaum.

Richey, R. C. (1998). The pursuit of useable knowledge in instructional technology. Educational Technology Research and Development, 46(4), 7-22.

Richey, R. C., Klein, J. D., y Nelson, W. (2004). Developmental research: Studies of instructional design and development. En Jonassen, D. (Ed.), Handbook of research for educational Communications and technology 2nd ed., (p. 1099-1130). Mahwah, NJ: Lawrence Erlbaum Associates, Publishers.

Richey, R. T., y Nelson, W. A. (1996). Developmental research. En Jonassen, D. (Ed.), Handbook of research for educational communications and technology (p. 1213-1245). New York: Macmillan.

Richey, R. y Klein, J. (2007). Design and Development Research: Methods, Strategies, and Issues. NY: Routledge

Rossi, M. and Sein, M. (2003). Design Research Workshop: A Proactive Research Approach. Presentation delivered at IRIS 26, August 9-12, 2003.

Salinas, J. (2012). La investigación ante los desafíos de los escenarios de aprendizaje futuros. RED. Revista de Educación a Distancia, 32. Recuperado de http://www.um.es/ead/red/32/salinas.pdf

Salinas, J.; Pérez, A. y de Benito, B. (2008). Metodologías centradas en el alumno para el aprendizaje en red. Madrid: Síntesis.

Shavelson, R. J., Phillips, D. C., Towne, L., y Feuer, M. J. (2003). On the science of education design studies. Educational Researcher, 32(1), 25-28. 
Simon, H. A. (1996). The sciences of the artificial (3rd ed.). Cambridge, MA: MIT Press.

Stokes, D. E. (1997). Pasteur's quadrant: Basic science and technological innovation. Washington, DC: Brookings Institution Press.

Van den Akker, J. (1999). Principles and methods of development research. En J. van den Akker, N. Nieveen, R. M. Branch, K. L. Gustafson, y T. Plomp, (Eds.), Design methodology and developmental research in education and training (pp. 1-14). The Netherlands: Kluwer Academic Publishers

Van den Akker, J. y Plomp, T. (1993). Development research in curriculum: Propositions and experiences. Paper presented at AERA meeting, April 12-16, Atlanta.

Walker, D. F. (1992). Methodological issues in educational research. En Jackson, P. W. (1992). (Ed.), Handbook of Research on Curriculum: A Project of the American Educational Research Association. New York: Macmillan.

Wang, F., y Hannafin, M. J. (2005). Design-based research and technology- enhanced learning environments. Educational Technology Research and Development, 53(4), 5-23.

\section{INFORMACIÓN SOBRE LOS AUTORES}

\section{Barbara de Benito Crosetti}

Universitat de les Illes Balears

Profesora contrada doctor (desde 2007) en el departamento de Pedagogía Aplicada i Psicología de la Educación de la UIB, impartiendo la asignatura de Tecnología Educativa I y Tecnología Educativa II de los estudios de pedagogía.

Miembro del Grupo de Tecnología Educativa (GTE) desde su creación en 1992 hasta la actualidad, donde ha participado en diferentes proyectos de investigación, de diseño de materiales y de análisis, diseño, implementación y evaluación de entornos virtuales de formación.

Experiencia en docencia de grado, postgrado y formación de formadores, tanto a nivel nacional como internacional.

\section{Jesus María Salinas Ibáñez}

Universitat de les Illes Balears

Doctor en Filosofía y Ciencias de la Educación. Catedrático de Tecnología Educativa de la Universitat de les Illes Balears. Investigador Principal del Grup de Tecnologia Educativa. Coordinador del Doctorado Interuniversitario en Tecnología Educativa. Director del Master en Tecnología Educativa: elearning y gestión del conocimiento. Director de Edutec Revista electrónica de Tecnología Educativa

\section{(cc) EY-NC}

Los textos publicados en esta revista están sujetos a una licencia de Reconocimiento 4.0 España de Creative Commons. Puede copiarlos, distribuirlos, comunicarlos públicamente y hacer obras derivadas siempre que reconozca los créditos de las obras (autoría, nombre de la revista, institución editora) de la manera especificada por los autores o por la revista. La licencia completa se puede consultar en:Licencia Creative Commons Atribución-NoComercial 4.0 Internacional. 